\title{
Kinetic arrest of crowded soft spheres in solvents of varying quality
}

\author{
E. Stiakakis, ${ }^{1}$ D. Vlassopoulos,,${ }^{1,2}$ B. Loppinet, ${ }^{1}$ J. Roovers, ${ }^{1,3}$ and G. Meier ${ }^{4}$ \\ ${ }^{1}$ FORTH, Institute of Electronic Structure and Laser, GR-71110 Heraklion, Crete, Greece \\ ${ }^{2}$ University of Crete, Department of Materials Science and Technology, GR-71003 Heraklion, Crete, Greece \\ ${ }^{3}$ NRC, Institute for Chemical Process and Environmental Technology, Ottawa, Ontario, Canada K1A OR6 \\ ${ }^{4}$ Forschungszentrum Jülich, Institut für Festkörperforschung, D-52425 Jülich, Germany
}

(Received 23 July 2002; published 13 November 2002)

\begin{abstract}
Crowded solutions of multiarm star polymers, representing model colloidal spheres with ultrasoft repulsive interactions, undergo a reversible gelation transition upon heating in solvents of intermediate quality (between good and $\Theta$ ). This unusual phenomenon is due to the kinetic arrest of the swollen interpenetrating spheres at high temperatures, forming clusters, in analogy to the colloidal glass transition. In this work we demonstrate that the choice of the solvent has a dramatic effect on the gelation transition, because of the different degree of star swelling (at the same temperature) associated with the solvent quality. We construct a generic kinetic phase diagram for the gelation of different stars in different solvents (gelation temperature against effective volume fraction, $\phi$ ) and propose a critical "soft sphere close packing" volume fraction $\phi_{c}$ distinguishing the temperature-induced (for $\phi<\phi_{c}$ ) from the concentration-induced (for $\phi>\phi_{c}$ ) glass-like gelation. We conclude that appropriate selection of the solvent allows for manipulation of the sol-gel transition in such ultrasoft colloids.
\end{abstract}

DOI: $10.1103 /$ PhysRevE.66.051804

PACS number(s): $61.25 . \mathrm{Hq}, 82.70 . \mathrm{Dd}, 82.70 . \mathrm{Gg}, 83.80 . \mathrm{Hj}$

\section{INTRODUCTION}

Colloidal gelation and glass transition have fascinated scientists for many years $[1,2]$. These intriguing physical phenomena relate to the phase behavior of colloidal dispersions, and consequently to colloidal stabilization, which is of great importance for a variety of applications ranging from food and biological products to oil recovery and coatings [3]. Whereas gelation and glass transition possess many common features, they also exhibit distinct differences originating at molecular level. It is thus of prime interest to understand thoroughly gelation, glass transition and in more general terms the physics of the transition from liquid to (amorphous) solid in colloidal suspensions. Till date, most of the work addressing these topics has been carried out with hard spheres [1,3-6], which have been found to undergo a glass transition to a disordered solid at a volume fraction $\phi$ $\sim 0.58$; this transition is manifested as a nondecaying component of the dynamic structure factor and implies a structural arrest of the crowded suspension [4-6]. More recently, soft colloids with weakly attractive interactions were investigated [2]. Remarkably, glass transition and gelation were found to be intrinsically related with the fluid to solid transition, which is manifested as a kinetic arrest, and is driven by the crowding of single particles (caging due to steric hindrance) or clusters of particles (attraction-driven), respectively $[2,7]$. These observations corroborate the notion of a generic jamming transition, a characteristic of a wide range of soft materials which lose their ability to flow at high volume fractions $[1,8,9]$.

Of particular interest in this class of materials, which become kinetically trapped when crowded, are polymerically stabilized colloids, such as colloidal particles with grafted polymeric chains, the latter representing the stabilizing medium $[10,11]$. In these systems both colloidal (core) and polymeric (chain) behavior contribute to the structure and dynamics of the suspension, and provide two groups of molecular parameters for tuning such liquid-solid transitions. Multiarm star polymers constitute a limiting case of this type of suspension, being characterized by a nonuniform monomer density profile, and yielding a very small deformable core and corona of grafted chains with a typical size about 10 times that of the core (see Fig. 1) $[12,13]$. They are known to represent model ultrasoft colloidal spheres with wide ranging weak repulsive potential [14], which is monitored by the number and size of the star arms. In these systems the polymeric and colloidal features have been identified and relate primarily to arm (or arm segment) collective relaxation and overall star self-diffusion, respectively [13]. Their interplay reflects the relative polymeric and colloidal contributions on the system's dynamics, suggesting ways for molecular design and control of such hybrid soft materials $[13,15,16]$.

It was shown experimentally, that when suspended in solvents of intermediate quality between good [17] and $\Theta$ [18] (characterized by excluded volume interactions only and neglecting the weak van der Waals attraction) at high volume fractions, these star polymers exhibited a dramatic increase in their mechanical moduli upon heating, eventually yielding a solid-like response [19]. This phenomenon was characterized by a slow kinetics of heating-induced gelation and an even slower kinetics of liquification upon cooling [20], and was attributed to the formation of clusters causing a partial dynamic arrest of the swollen interpenetrating spheres at high temperatures. A kinetic phase diagram was proposed and discussed in analogy to the phase diagram of chargestabilized colloids; in the latter case colloids exhibited macrocrystalline order due to the increase of the number concentration, whereas the present ultrasoft systems gelled at constant number of stars. Thus, achieving (through different means) a high enough volume fraction (corresponding to overlapping of the spheres) is the prerequisite for gelation.

According to the proposed mechanism of the reversible 


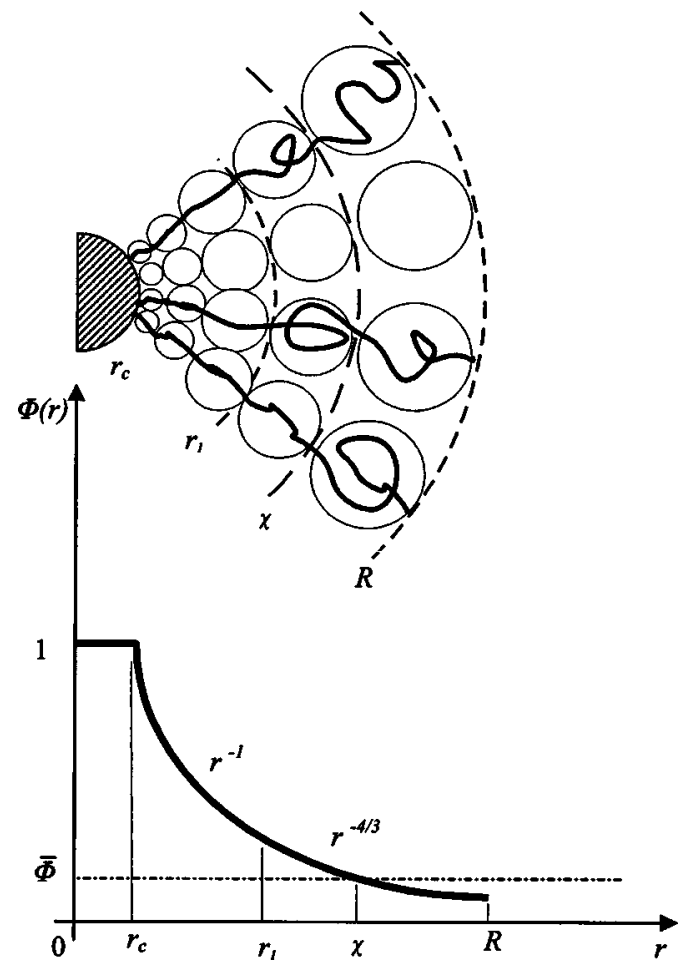

FIG. 1. Schematic representation of the structure of a regular multiarm star polymer (top) and its nonuniform monomer density distribution (with different scaling regimes), $\Phi(r)$, according to the Daoud-Cotton model [12] (bottom). Dashed circles attempt to illustrate the overall spherical shape of these macromolecules. The three characteristic radii $r_{c}, r_{1}$, and $R$, define the melt-like, $\Theta$ solventlike $\left[\Phi(r) \sim r^{-1}\right]$, and excluded volume regions $\left[\Phi(r) \sim r^{-4 / 3}\right]$, respectively. The value of $r=\chi$ defines the radial distance of the single star in semidilute solution, beyond which there is a screening of the interaction (because of the mutual interpenetration of different stars, which are not drawn here) and the size of the blob is controlled by the average solution concentration $\bar{\Phi}$.

thermal gelation in intermediate solvents $[19,20]$, as the temperature increases the solvent quality improves and the peripheral blobs of the interacting liquid-ordered soft spheres swell; consequently, the spheres enhance their arms' overlap, but not to a large extent because of an increasingly strong excluded volume repulsion. This crowding eventually leads to a kinetic frustration (jamming) of the stars due to the formation of clusters consisting of trapped spheres (about half of the total population of spheres) in equilibrium with "free" spheres $[19,20]$.

The star chain expansion upon heating is responsible for the slow formation of long lived clusters (involving some kind of cooperativity) which kinetically arrest the system and form a gel, much like a glass formation process. This represents a generic behavior for this class of soft materials, for which the size ratio of "grafted" layer to central core is about 10 times larger than in most of the "conventional" block copolymer micelles or sterically stabilized colloids used so far [21-25]. Note that a recently reported thermoreversible gelation observed with aqueous dispersions of polystyrene latex particles bearing grafted poly(ethylene oxide) chains [26] is clearly different from the present situa-
TABLE I. Molecular characteristics of the star polymers.

\begin{tabular}{lcccc}
\hline \hline Sample & $\mathrm{f}$ & $M_{a}(\mathrm{~g} / \mathrm{mol})$ & $R_{h}(\mathrm{~nm})^{\mathrm{a}}$ & $c^{*}(\mathrm{mg} / \mathrm{ml})^{\mathrm{b}}$ \\
\hline 12880 & 122 & 72100 & $48.5(45)^{\mathrm{c}}$ & $30.6(38.3)^{\mathrm{c}}$ \\
12856 & 127 & 47300 & 37.8 & 45.1 \\
12828 & 114 & 26100 & 26.1 & 73.0 \\
\hline \hline
\end{tabular}

${ }^{\mathrm{a}}$ From dynamic light scattering measurements in decane, at $20^{\circ} \mathrm{C}$ [19].

${ }^{\mathrm{b}}$ Estimated (in decane) from the hydrodynamic radius at $20^{\circ} \mathrm{C}$, $c^{*}=\left[(4 \pi / 3) R_{h}^{3}\right]^{-1}\left(f M_{a} / N_{A}\right)$.

${ }^{\mathrm{c}}$ The value in parentheses refers to tetradecane at $20^{\circ} \mathrm{C}$.

tion; in that work, the (short) chains form a lower critical solution temperature system in water, and gelation was attributed to attractive interactions (of van der Waals and osmotic origin), which resulted in a weak secondary minimum of the interparticle potential. In addition to the above, the present findings demonstrate how the effective volume fraction of these crowded soft systems can increase with temperature apart from density, offering a new route to reversible gelation and an alternative means for jamming of soft matter $[1,9,19,20]$.

In this paper we investigate the effects of solvent quality. By switching from decane to tetradecane, the solvent quality decreases as evidenced by dilute solution measurements. This has a dramatic impact on shifting the gelation concentration and temperature to higher values. These effects as well as the proposed generic kinetic phase diagram for different stars and solvents, along with its implications for colloidal jamming transitions, are discussed in the following sections. Therefore, this work contributes toward a unified description of the glass-like gelation in ultrasoft polymeric spheres and its molecular manipulation.

\section{EXPERIMENT}

\section{A. Materials}

Using a dendrimer scaffold and chlorosilane coupling chemistry, living linear 1,4-polybutadiene arms were assembled into regular stars with nominal functionality $f$ $=128$ and nominal arm molecular weight $M_{a}$ $=80000 \mathrm{~g} / \mathrm{mol}$, coded as 12880 [27]. For comparison, data obtained with similar stars of different arm molecular weight (28 000 and $56000 \mathrm{~g} / \mathrm{mol}$, coded as 12828 and 12856 , respectively) were also used [27]. These chemically homogeneous soft materials are spherical in shape (see illustration in Figs. 1 and 2), and are called regular stars in order to distinguish them from their irregular counterparts [20], which are not considered in this work. They exhibit a nonuniform segment density distribution with a higher density in the core region as predicted theoretically and confirmed experimentally (Fig. 1) $[14,28,29]$. Moreover, above the overlap concentration they are characterized by liquid-like ordering (due to the enhanced osmotic pressure gradient), a soft interaction potential, and a resulting rich dynamic response [1113,28,29].

The actual molecular characteristics of the three multiarm star polymers considered in this work are listed in Table I. 
The polymers were mixed with tetradecane, a solvent of "intermediate" quality (as discussed below) for 1,4polybutadiene, followed by gentle stirring at $20^{\circ} \mathrm{C}$ for at least $8 \mathrm{~h}$ (conditions identical to the decane case). A small amount (0.1 wt\%) of antioxidant (4-methyl-2,6-di-tertbutylphenol) was added to prevent degradation.

\section{B. Methods}

The dilute solution characterization, as well as the dynamics and structure of the crowded multiarm star polymer solutions were investigated at different temperatures using shear rheology, small angle neutron scattering (SANS) and dynamic light scattering.

A rheometric scientific ARES-HR strain-controlled rheometer was utilized with a force rebalance transducer 100FRTN1 in the parallel plate geometry (diameter $25 \mathrm{~mm}$, sample gap about $1 \mathrm{~mm}$ ). Temperature control in the range of $\pm 0.1^{\circ} \mathrm{C}$ was achieved via a recirculating ethylene glycol/ water mixture for temperatures between -10 and $70^{\circ} \mathrm{C}$. Measurements included dynamic frequency sweeps and temperature ramps (to determine the gelation temperature), dynamic strain sweeps (to establish the limits of the linear viscoelastic response) and dynamic time sweeps (to establish steady state measurement conditions).

The measurements of the effective hydrodynamic radii of the single stars (in dilute solution) were carried out with dynamic light scattering by utilizing an ALV goniometer setup and an ADLAS DRY-325 Nd:YAG laser operating at a power of $100 \mathrm{~mW}$ and a wavelength $\lambda=532 \mathrm{~nm}$. The Brownian motion of the polymer was detected through the concentration fluctuations of the system at different scattering wave vectors $q=(4 \pi n / \lambda) \sin (\theta / 2), n$ being the refractive index of the solvent and $\theta$ the scattering angle. The time autocorrelation function of the scattered intensity $G(q, t)$ was determined with the aid of an ALV-5000/E fast multi- $\tau$ correlator in the time range $10^{-7}-10^{3} \mathrm{~s}$. The measurement consisted of obtaining the intermediate scattering (field) function $C(q, t)=\left[(G(q, t)-1) / f^{*}\right]^{1 / 2}$ in the polarized (VV) geometry, where $f^{*}$ is an instrumental factor relating to the coherence area [30]. The equivalent hydrodynamic radius was extracted from the measured diffusion coefficient assuming validity of the Stokes-Einstein relation, $R_{h}$ $=k T / 6 \pi \eta D$ ( $k$ being the Boltzmann's constant, $T$ the temperature, and $\eta$ the solvent viscosity) for spherical objects.

SANS experiments were performed at the KWS II, FZJülich, using neutron wavelength $\lambda=6.32 \AA$ with $\Delta \lambda / \lambda$ $\approx 0.18$ and three detector distances $(2,8$, and $20 \mathrm{~m})$ to access the whole scattering wave vector range $\left[0.03<q\left(\mathrm{~nm}^{-1}\right)\right.$ $<1.5)$. The scattered neutrons were detected with a twodimensional ${ }^{3} \mathrm{He}$ detector consisting of $64 \times 64$ channels, each of $0.8 \mathrm{~cm}$ width. The count rate was controlled in such a way that dead time effects were negligible. The isotropic raw data were radially averaged, corrected for background scattering, and the intensity was converted into absolute units $\left(\mathrm{cm}^{-1}\right)$ by using a polyethylene sample of known incoherent scattering as standard (calibrated against vanadium) and employing the Jülich software package DAN. Solutions in perdeuterated tetradecane (with $0.1 \% \mathrm{wt}$ antioxidant) were mea-

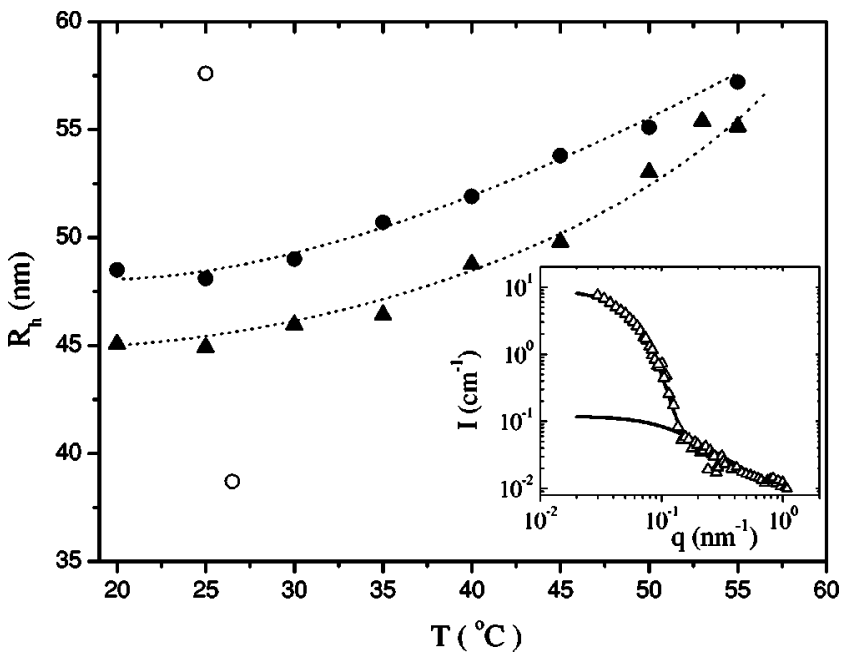

FIG. 2. Temperature dependence of the effective hydrodynamic radius $R_{h}$ of 12880 in tetradecane $(\boldsymbol{\Delta})$ and decane $(\boldsymbol{O})$, as determined from dynamic light scattering measurements in dilute solutions $\left(c<c^{*}\right)$. The lines are drawn to guide the eye. The open circles $(\bigcirc)$ refer to the values in a good (nearly athermal) solvent cyclohexane at $25^{\circ} \mathrm{C}$ (high value) and a theta solvent dioxane at $26.5^{\circ} \mathrm{C}$ (low value). Inset: double logarithmic $\left(\log _{10}\right)$ plot of the SANS total scattering intensity against the scattering wave vector from dilute solution of $12880(0.1 \mathrm{wt} \%)$ in $d$-tetradecane at $20^{\circ} \mathrm{C}$ $(\Delta)$. Lines are fits to the different $q$-regimes (see text).

sured in $1 \mathrm{~mm}$ thick Hellma quartz cuvettes, heated to the desired temperatures (where the sample was equilibrated for more than $1 \mathrm{~h}$ ) with an accuracy of $\pm 1{ }^{\circ} \mathrm{C}$.

\section{RESULTS AND DISCUSSION}

\section{A. Dilute solution characterization}

Figure 2 depicts the hydrodynamic radius of 12880 in tetradecane as function of temperature. For comparison, the respective data for the "better quality" solvent decane are shown, as well as the values of the radius in a good (nearly athermal) solvent (cyclohexane, measured at $25^{\circ} \mathrm{C}$ ) and a theta solvent (dioxane, measured at the theta temperature $\Theta$ $=26.5^{\circ} \mathrm{C}$ ) [27]. All measurements were performed in dilute solutions (concentrations in the range of $0.01 \mathrm{wt} \%$ ).

The significant swelling observed upon heating (about $20 \%$ in the temperature range studied) suggests that tetradecane is not a truly good solvent near room temperature, and only approaches good solvent (e.g., cyclohexane) behavior above $55^{\circ} \mathrm{C}$. On the other hand, it is evident that tetradecane is a solvent of reduced quality for 1,4-polybutadiene compared to decane, as the $\mathrm{R}_{h}$ values of the stars are consistently lower in the former case. These findings suggest that these hydrocarbons are solvents of intermediate (or moderate) quality, exhibiting a strong temperature dependence in the range investigated, and approaching the $\Theta$ conditions as the number of carbons is increased. In the discussion below we use $\mathrm{R}_{h}$ as an index of star dimension. Whereas this is a dynamic length, given the fact that for high functionality stars the hydrodynamic solvent draining is not too strong [27,31] (and thus it should not be much influenced by tem- 
perature or concentration), we argue that the observed effects relate to star size changes.

The inset of Fig. 2 depicts the SANS scattering intensity vs $q$, measured in dilute solution of $12880(0.1 \mathrm{wt} \%)$ in $d$-tetradecane at $47^{\circ} \mathrm{C}$. It can be observed that the data exhibit the typical scattering behavior of multiarm stars, with two apparent distinct contributions at small and large scattering wave vector values. The low- $q$ part $\left(q R_{g} \ll 1, R_{g}\right.$ being the radius of gyration) is dominated by the "form factor" scattering due to the overall polymer density profile, whereas the high- $q$ part is dominated by the polymer-solvent fluctuations (blob term). This leads to the representation of the SANS intensity by two independent contributions $[28,29,32-34]$

$$
I(q)=I(0) e^{-q^{2} R_{g}^{2} / 3}+\frac{4 \pi \alpha}{q \xi} \frac{\sin \left[(2 / 3) \tan ^{-1}(q \xi)\right]}{\left[1+q^{2} \xi^{2}\right]^{1 / 3}} \Gamma\left(\frac{2}{3}\right) .
$$

In this equation, $\xi$ is a correlation length (typically it is taken as the diameter of the outermost blobs, $\xi=2 \mathrm{R}_{g} / \sqrt{f}$ ). The first contribution in the right-hand side of this equation is the simplest expression of the form factor (Guinier type) [33]. The second term (high- $q$ limit) is the Dozier et al. expression [33], where $\Gamma$ denotes the gamma function and $\alpha$ is a normalization constant, treated in this analysis as an adjustable parameter, along with $R_{g}, \xi$ and $I(0)$.

The best fit of the above equation for the 12880 at $47^{\circ} \mathrm{C}$, is shown in the inset of Fig. 2, and is very satisfactory; the high- $q$ data appear to conform to the $q^{-5 / 3}$ scaling [Eq. (1)] for good solvent $[18,20,28,34]$. The obtained value of $R_{g}$ $\approx 32 \mathrm{~nm}(\xi \approx 5.7 \mathrm{~nm})$ is known to be underestimated, primarily because of the insufficient low- $q$ limit (yielding $q R_{g}$ $\approx 1$; in fact, the range $q R_{g}<1$ should be reached for an accurate determination of $R_{g}$ ). Alternatively, $R_{g}$ can be obtained from the maximum of a modified Kratky plot $\left(I q^{1.67}\right.$ vs $q$ ), yielding a respective value of $36 \mathrm{~nm}(\xi \approx 6.5 \mathrm{~nm})$. In cyclohexane, a good solvent, the reported value of $R_{g}$ from light scattering measurements (at $20^{\circ} \mathrm{C}$ ) is $42.4 \mathrm{~nm}$ [27]. For this case, the ratio $R_{h} / R_{g}$ is about 1.4 , in agreement with similar studies with other multiarm stars in various solvents $[13,27,28]$.

\section{B. Behavior in dense solutions}

The dramatic effect of temperature in inducing a liquidsolid transition is clearly demonstrated in the rheological data of Fig. 3 for a $5 \mathrm{wt} \%$ solution of 12880 in tetradecane. At lower temperatures $\left(40\right.$ and $\left.50^{\circ} \mathrm{C}\right)$ the polymer solution remains a viscous liquid (terminal behavior with $\mathrm{G}^{\prime} \sim \omega^{2}$, $\left.\mathrm{G}^{\prime \prime} \sim \omega, \mathrm{G}^{\prime \prime}>\mathrm{G}^{\prime}\right)$, whereas at a higher temperature $\left(55^{\circ} \mathrm{C}\right)$ it is reversibly transformed into a weak elastic solid $\left(\mathrm{G}^{\prime}>\mathrm{G}^{\prime \prime}\right.$ and a very weak frequency dependence over more than three decades in frequency), accompanied by a sharp rise in the values of the moduli with respect to the liquid. The reproducibility of these results at different times following cooling to room temperature and recovering the original liquid, confirms the reversibility of the process and is consistent with visual observations of this transition, as well as respective

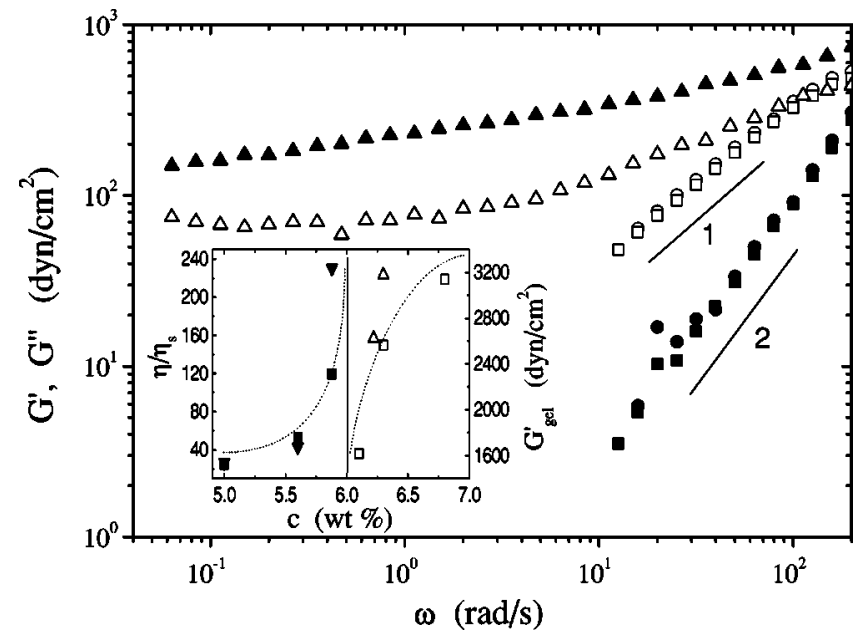

FIG. 3. Double logarithmic $\left(\log _{10}\right)$ plot depicting the dynamic frequency sweeps of $G^{\prime}$ (closed symbols) and $G^{\prime \prime}$ (open symbols) for $128805 \mathrm{wt} \%$ in tetradecane at different temperatures across the gelation transition $\left(\boldsymbol{O}: 40^{\circ} \mathrm{C} ; \boldsymbol{\square}: 50{ }^{\circ} \mathrm{C} ; \boldsymbol{\Delta}: 55^{\circ} \mathrm{C}\right)$. The gelation transition takes place from 50 to $55^{\circ} \mathrm{C}$. Inset: Concentration dependence of reduced zero-shear viscosity (left, bold symbols) and elastic plateau modulus (right, open symbols) for two different temperatures $\left(40{ }^{\circ} \mathrm{C}: \boldsymbol{\nabla}, \triangle ; 30^{\circ} \mathrm{C}: \boldsymbol{\square}, \square\right)$. The solid vertical line identifies the critical concentration for sol-gel transition. The dotted lines are drawn to guide the eye.

data obtained in decane solutions [20]. The inset of Fig. 3 depicts the concentration dependence of the reduced (to the solvent) zero-shear viscosity (in the liquid regime, on the left) $\eta / \eta_{s}$ and plateau modulus (in the gel-like regime, on the right) $G_{g e l}^{\prime}$ at two different temperatures. It is evident that there is a certain critical concentration, where both viscosity and plateau modulus diverge; this concentration (about 6 wt $\%$ here) signifies the reversible sol-gel transition. This type of representation identifies the control parameters for gelation and is more general, as it describes a wide range of jammed solids $[8,9,35]$. According to this figure, there is a minimum concentration above which the star suspension remains always in the gel state (in the concentration range examined). Despite the limited data with small variation (note the linear scale), it appears that both viscosity and elastic plateau modulus diverge at a finite critical concentration, bringing analogies to critical behavior observed in other colloidal systems [8,36-38].

A kinetic pseudophase diagram of the gelation temperature $T_{g e l}$ as a function of star polymer concentration $c$ is presented in Fig. 4(a). In this figure the respective data for the $12880 /$ decane system are also shown for comparison. It is evident even a small change in the solvent quality (through a variation of the molecular weight while maintaining the same chemical constitution) has a dramatic impact on the behavior of this ultrasoft suspension, as it shifts the gelation temperature and concentration to much higher values. The respective plateau moduli at $T_{g e l}, \mathrm{G}_{g e l}^{\prime}\left(T_{g e l}\right)$, are depicted in Fig. 4(b). It is interesting that $\mathrm{G}_{g e l}^{\prime}$ for the two solvents (corresponding to different $T_{g e l}$ ) are essentially identical, suggesting a generic effect of the solvent quality, as will be discussed below. The most remarkable effect from Fig. 4 


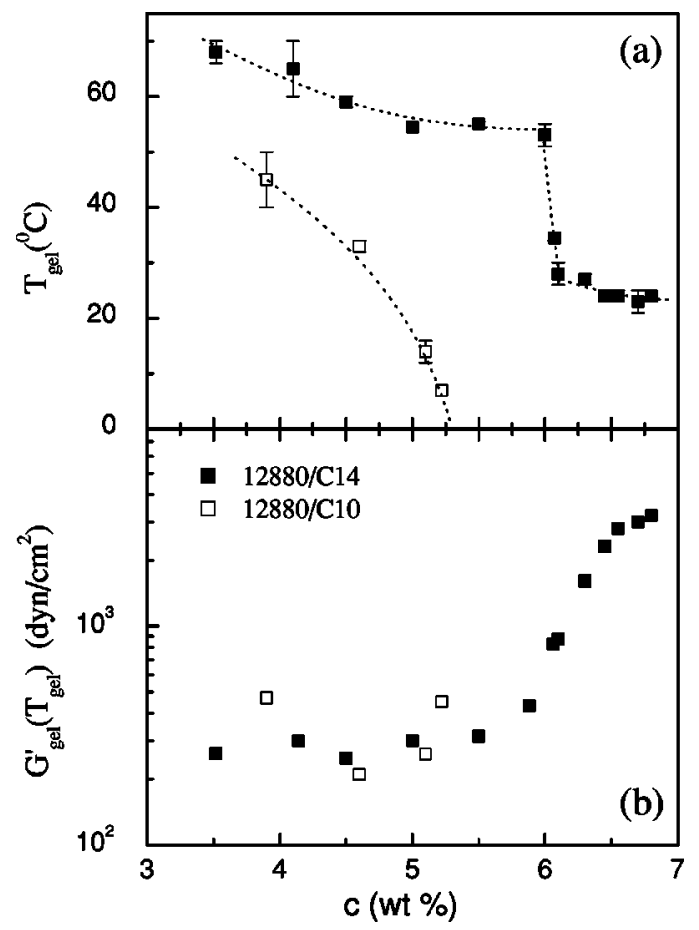

FIG. 4. (a) Gelation temperature $T_{g e l}$ as function of polymer concentration $c$ for the 12880 stars in two solvents: tetradecane $(\square)$ and decane ( $\boldsymbol{\square})$. Lines are drawn to guide the eye. (b) Respective concentration dependence of the plateau moduli $G_{g e l}^{\prime}\left(T_{g e l}\right)$ at $T_{\text {gel }}$ [semi-logarithmic $\left(\log _{10}\right)$ plot].

however, is the dramatic change of both $T_{g e l}$ and $\mathrm{G}_{g e l}^{\prime}\left(T_{g e l}\right)$ over a very small concentration range, indicating that with increasing concentration the ability to form gels is enhanced and at the same time there is a transition from softer to stronger gels; these aspects will be elucidated below.

Therefore, it is evident that the solvent itself can serve as an effective means to manipulate the sol-gel transition under otherwise constant conditions. As a demonstration, Fig. 5

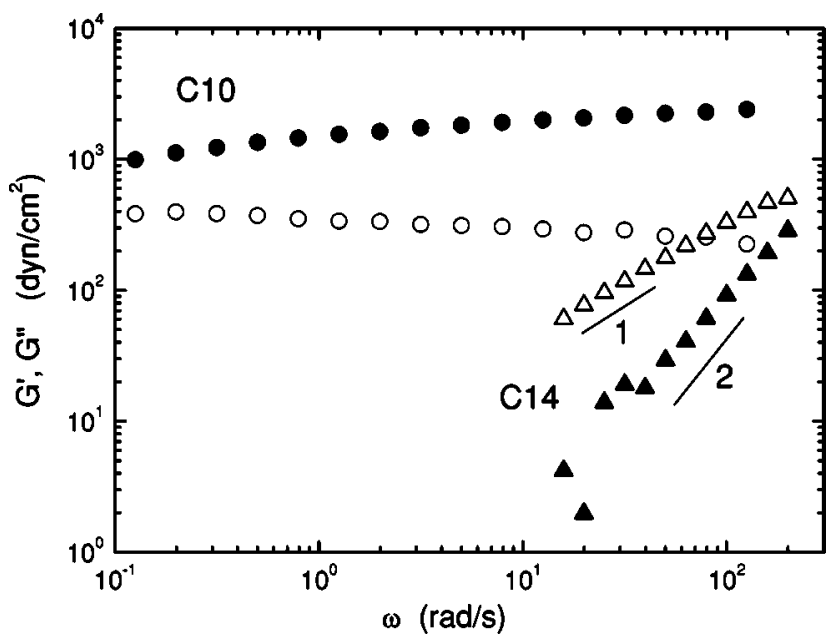

FIG. 5. Double logarithmic $\left(\log _{10}\right)$ plot of the linear viscoelastic spectra (solid symbols for $G^{\prime}$ ) of two solutions of $128805 \mathrm{wt} \%$ at the same temperature $T=40^{\circ} \mathrm{C}$, in two different solvents, tetradecane $(\mathrm{C} 14, \boldsymbol{\Delta})$ and decane $(\mathrm{C} 10, \mathbf{O})$.

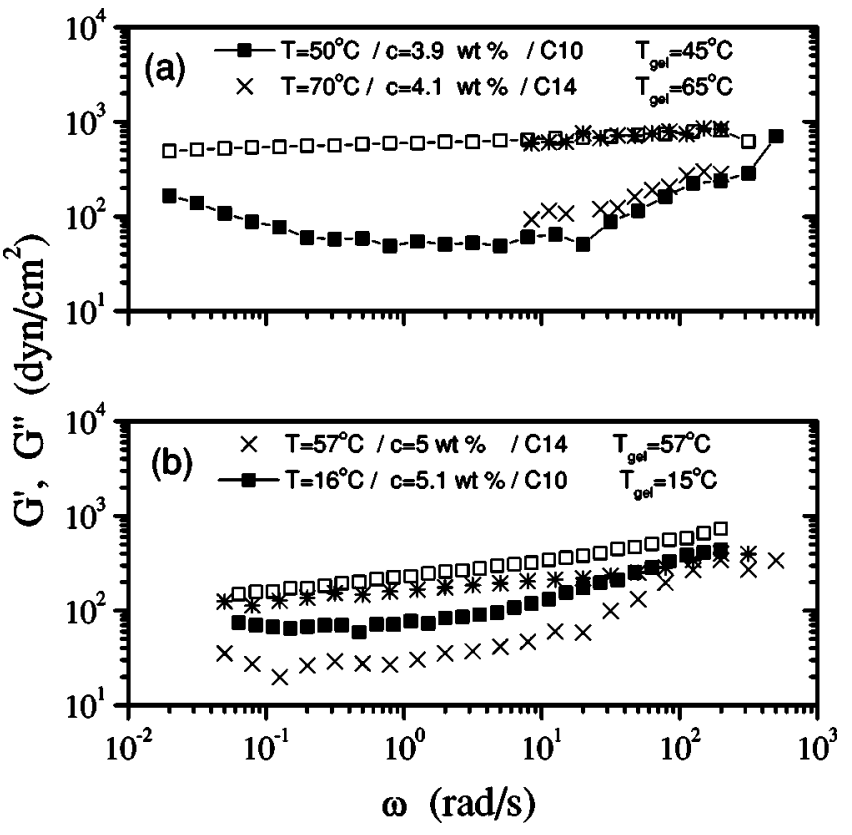

FIG. 6. Double logarithmic $\left(\log _{10}\right)$ plot depicting the linear viscoelastic spectra of 12880 gels $\left(G^{\prime}>G^{\prime \prime}\right)$ in decane ( $C 10$, squares) and tetradecane $(C 14$, crosses) of virtually the same concentration and distance from the gelation temperature $\Delta T=T-T_{\text {gel }}$. (a) $c$ $\approx 4 \mathrm{wt} \%, \Delta T=5{ }^{\circ} \mathrm{C} ;(\mathrm{b}) c \approx 5 \mathrm{wt} \%, \Delta T \approx 0{ }^{\circ} \mathrm{C}$.

depicts the linear viscoelastic spectra of two 12880 solutions of nearly the same concentration $c=5 \mathrm{wt} \%$, at the same temperature $T=40^{\circ} \mathrm{C}$ and in two different solvents, tetradecane (liquid behavior) and decane (solid behavior); this is a reflection of the interplay between solvent quality and temperature. The implication from this figure is that, much like in the case of thermodynamic transitions, the material properties depend on the distance from the phase boundary. Indeed, Fig. 6 confirms this point, by displaying the (nearly identical) linear viscoelastic spectra of two different gels in the two solvents at (virtually) the same concentration and a constant distance from the gelation temperature $\Delta T=T$ $-T_{g e l}$. Therefore, to achieve certain properties at a given concentration, one can control the temperature $T_{\text {gel }}$ through variation of the solvent.

The shape of the tetradecane phase boundary in Fig. 4(a), for which a much more detailed investigation was undertaken compared to the decane case, is quite remarkable. Starting from the less dense solutions, $T_{\text {gel }}$ exhibits a weak decrease with concentration up to a critical value where it drops sharply; beyond this concentration, $T_{g e l}$ is practically insensitive to concentration. In Fig. 7 we show an attempt to obtain a universal kinetic phase diagram by plotting in $T_{g e l}$ against the effective volume fraction of the stars, $\phi_{\text {eff }}$; the latter quantity is the volume fraction of the spherical stars by considering their overall dimension as if they were hard spheres, and is determined as $c / c^{*}$ where $c^{*}$ $=\left(4 \pi / 3 R_{h}^{3}\right)^{-1} f M_{a} / N_{A}$ [39] (with $R_{h}$ referring to a temperature of $20^{\circ} \mathrm{C}$, in the temperature-independent region). The dramatic drop in $T_{g e l}$ beyond a critical value of $\phi_{e f f}$, called $\phi_{c}$, is evident. In this figure, data from different multiarm stars in decane [19] are also included; even for these data 


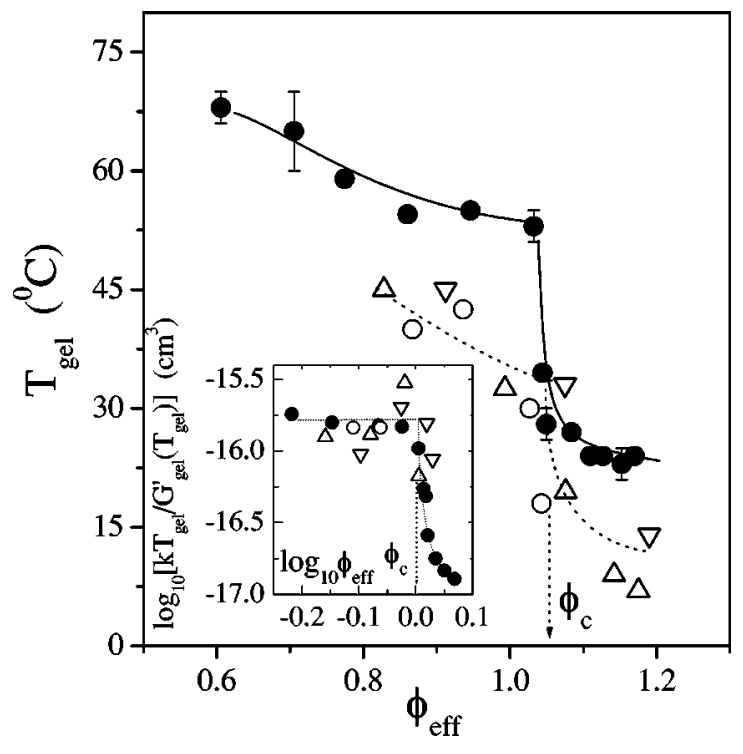

FIG. 7. Gelation temperature $T_{g e l}$ as function of the effective volume fraction $\phi_{\text {eff }}$ for different starts in different solvents: 12828 in decane $(\bigcirc) ; 12856$ in decane $(\triangle) ; 12880$ in decane $(\nabla) ; 12880$ in tetradecane $(\bigcirc)$. Lines are drawn to guide the eye. The vertical arrow indicates the critical volume fraction $\phi_{c}$ (see text). Inset: Double logarithmic representation of the effective interaction volume $\left[k T_{g e l} / G_{g e l}^{\prime}\left(T_{g e l}\right)\right]$ as function of the effective volume fraction $\left(\phi_{e f f}\right)$ for different stars in different solvents: 12828 in decane $(\bigcirc)$; 12856 in decane $(\triangle) ; 12880$ in decane $(\nabla) ; 12880$ in tetradecane $(\bigcirc)$. Lines are drawn to guide the eye.

which are more limited (per star) compared to the tetradecane case and exhibit a larger uncertainty (of about $\pm 3{ }^{\circ} \mathrm{C}$ ), a transition of $T_{g e l}$ can be observed at about the same value of $\phi_{c}$. Whereas this is a remarkable finding, this type of representation is specific for each solvent; apparently, the low- $\phi_{\text {eff }}$ data in the two solvents are separated by about $18^{\circ} \mathrm{C}$; this is due to the thermodynamic difference of the two solvents and corresponds roughly to the needed temperature shift for obtaining the same single star hydrodynamic size (see Fig. 2).

Alternatively, one can plot the quantity $\mathrm{k} T_{g e l} / G_{g e l}^{\prime}\left(T_{g e l}\right)$, which is an effective volume of interacting spheres in concentrated solution $\left(V_{i n t} \sim \sigma^{3}\right.$, with $\sigma$ being a typical size) giving rise to the stress in the gel state, against $\phi_{e f f}$, as seen in the inset of Fig. 7; the data for different stars and different solvents seem to follow the same trend for the lower concentration region. Taking the case of the thoroughly studied $12880 /$ tetradecane system, the experimental values of $V_{\text {int }}$ vary from $1.8 \times 10^{-16} \mathrm{~cm}^{3}$ in the lower $\phi_{\text {eff }}$ values to about $1.5 \times 10^{-17} \mathrm{~cm}^{3}$ in the higher $\phi_{\text {eff }}$ values. For stars with volume fraction exceeding the overlapping one, $\phi>\phi^{*}$, as in the present case, there is a region of space with size $\chi$ separating the single star behavior (for $\mathrm{r}<\chi$ ) from the semidilute solution behavior due to the screening of the interactions in the area of overlapping (for $r>\chi$ ) [12] (see also Fig. 1). For intermediate solvents, $\chi \sim\left(c l^{3}\right)^{-3 / 4} v^{-1 / 4} f^{1 / 2} l$ and the respective concentration blob size is $\xi(c) \sim\left(c l^{3}\right)^{-3 / 4} v^{-1 / 4} l[12]$. With an excluded volume parameter $v \approx(1-\Theta / T), \Theta$ being the theta temperature, it is clear from these expressions that as $\phi_{e f f}$ increases, and $T_{g e l}$ decreases [Figs. 4(a) and 7], the effective volume of the spheres $V_{\text {int }}$ will decrease if $\chi$ and $\xi(c)$ will decrease. This depends on both $v$ and $c$ and can be realized as long as there is swelling $\left(\phi<\phi_{\text {eff }}\right)$. For high concentrations $\phi>\phi_{\text {eff }}$ there is practically no swelling and concentration effect on $\chi$ for these very high functionality stars, and we are in a some kind of jamming region (which is not considered in the Daoud-Cotton model) [12], resembling (but clearly not being identical to) a melt-like behavior where $\chi \sim(f)^{1 / 2} l$, where $V_{\text {int }}$ (and $T_{g e l}$ ) is practically independent of $\phi$. The reason for this lies in the fact that the stars shrink and expand less with temperature as the concentration increases, which is offset by the increasing concentration itself (a kind of cage effect). This simple argument can rationalize the experimental findings of Fig. 7.

Based on the phenomenological evidence presented, we propose that the critical volume fraction corresponding to the drop of the value of $\mathrm{k} T_{g e l} / G_{g e l}^{\prime}\left(T_{g e l}\right)$ (by more than one decade) is an effective close packing volume fraction for soft spheres $\phi_{c}$ which separates this jamming phase diagram in two regions: the region $\phi_{e f f}<\phi_{c}$ where temperature-induced gelation takes place, and the region $\phi_{e f f}>\phi_{c}$ which is dominated by the concentration-induced gelation (see also the inset of Fig. 3). In the former case, the stars are less crowded and temperature induces swelling which is compensated by an enhanced ovelap $[19,20]$. In the latter very crowded case however, swelling is hindered by the effective cages formed by the star neighbors, which tend to practically compress the star, yielding a packing of the stars in cages, known as jamming; this is analogous to hard sphere colloidal glass, but with different origin. The transition around $\phi_{c}$ is quite sharp as there is a critical concentration of the stars undergoing swelling for forming the cages, as in glass transition. The extracted value of $\phi_{c}$ from Fig. 7 is about 1.05, and considered reasonable as it is clearly above that of hard spheres (around 0.69) due to partial interpenetration and squeezing of the crowded stars.

Note that the interaction volume depends primarily on $G_{g e l}^{\prime}\left(T_{g e l}\right)$; for $\phi_{e f f}<\phi_{c}$ it assumes a value about ten times smaller compared to the $\phi_{e f f}>\phi_{c}$ region, the latter being characterized by much stronger gels. These two distinct mechanisms across $\phi_{c}$ are also manifested in the phase diagram of the 12880/tetradecane system [Figs. 4(a) and 7], where in the former case a (weak) concentration dependence of $T_{g e l}$ is observed (as the higher the concentration, the "easier" it is to achieve gelation), whereas in the latter case $T_{g e l}$ is practically independent of concentration (since the system is very dense). Moreover, in the former case, the stars clearly swell upon heating and some random local clusters build-up (as evidenced by dynamic light scattering), forming the gel $[19,20]$; on the other hand, in the latter case, star swelling is probably very weak, and the dominant effect is that of concentration, giving rise to some kind of jamming. In this dense suspension, instead of only a few clusters being formed, the system rather builds several larger clusters that effectively could span the whole volume [not necessarily in the form of an interconnected network [40]]. This can rationalize the large difference (here amounting to a decade) of 


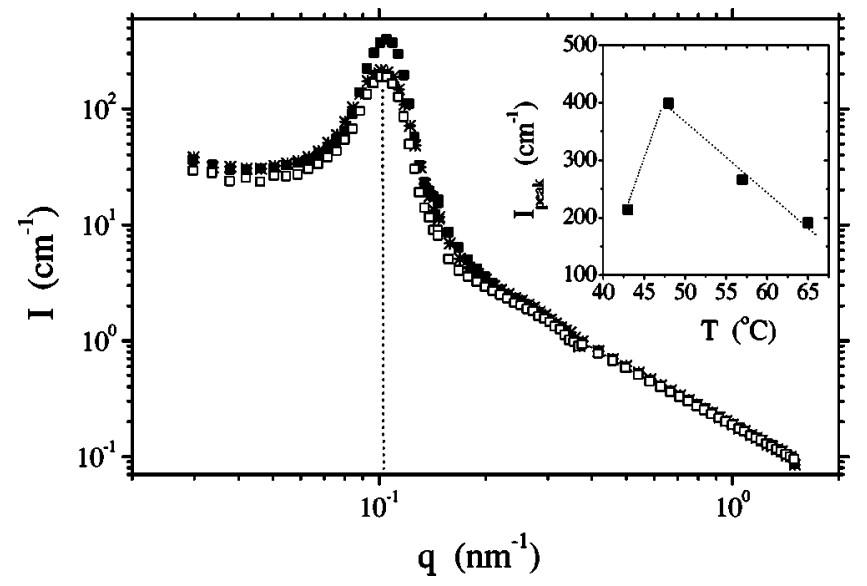

FIG. 8. Double logarithmic $\left(\log _{10}\right)$ plot depicting the SANS scattering intensity $I$ versus the scattering wave vector $q$ for 5.47 wt $\% 12880$ in $d$-tetradecane and three temperatures, $43^{\circ} \mathrm{C}$ (*; liquid), $48^{\circ} \mathrm{C}$ ( $\square$; liquid) and $65^{\circ} \mathrm{C}(\square$; gel). Inset: Temperature dependence of the peak intensity $I_{\text {peak }}$. Lines are drawn to guide the eye.

$G_{g e l}^{\prime}\left(T_{g e l}\right)$ across $\phi_{c}$. A qualitative analogy to jammed colloidal particles with attractive interactions can be made here, with respect to their elastic moduli, associating the temperature-induced gels to colloidal glasses and the concentration-induced gels to colloidal gels $[6,9,41]$.

In the above discussion the effective volume fraction was estimated from the hydrodynamic radius in the low temperature region, in order to account for the temperature-induced swelling in Fig. 7. If, on the other hand, one uses $\phi_{e f f}\left(T_{g e l}\right)$ based on $R_{h}\left(T_{g e l}\right)$, which essentially "absorbs" the swelling effect, one finds two virtually constant values of $\phi_{e f f}\left(T_{g e l}\right)$, namely 1.7 for $\phi_{e f f}<\phi_{c}$ and 1.1 for $\phi_{e f f}\left(T_{g e l}\right)>\phi_{c}$. Again, the distinction of the two gelation regimes is evident.

This distinction is also supported by structural studies carried out by SANS from solutions in deuterated tetradecane. The scattering intensities for two concentrations, namely $5.47 \mathrm{wt} \%$ below $\phi_{c}$ (Fig. 8) and $6.44 \mathrm{wt} \%$ above $\phi_{c}$ (Fig. 9) show the typical profile of interacting stars, with a sharp maximum (at $q_{\text {peak }}$ ) followed by a power law decay. The characteristic length $d \approx 2 \pi / q_{\text {peak }}$ is about $63 \mathrm{~nm}$ for both samples and independent of temperature, showing the strong stars interpenetration. The important finding here is the variation of the intensity at the maximum $\left(q_{\text {peak }}\right)$ when crossing the gelation boundary (insets of Figs. 8 and 9). We relate the intensity at the maximum $\left(I_{\text {peak }}\right)$ to the intensity of the structure factor $S\left(q_{\text {peak }}\right)$, neglecting the variation of form factor, which is expected to be very small for so small variation of temperature $[18,20]$. For the $5.47 \mathrm{wt} \%$ solution, $I_{\text {peak }}$ is found to increase with increasing temperature in the liquid state, as expected for a star solution in a solvent whose quality is improving (see also Fig. 2) [18]; this is a sign of better ordering resulting from stronger excluded volume interactions. However, with further increase of the temperature getting to the gel state, the intensity is no longer increasing but is found to decrease. This relates to stronger star interpenetration $[19,20]$ and represents a manifestation of how the gelation alters the ordering. On the other hand, for the more

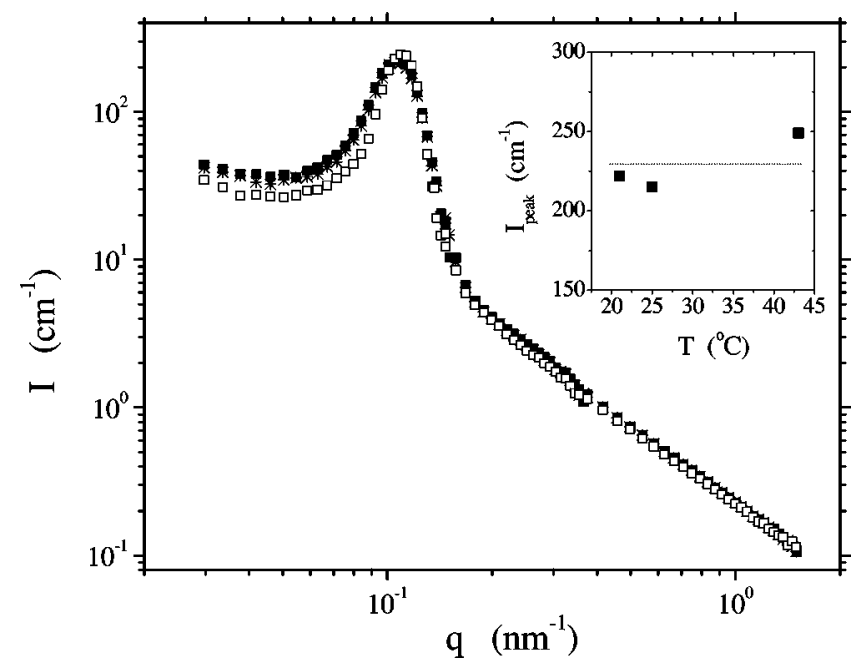

FIG. 9. Double logarithmic $\left(\log _{10}\right)$ plot depicting the SANS scattering intensity $I$ versus the scattering wave vector $q$ for 6.44 wt $\% 12880$ in $d$ tetradecane and three temperatures, $21^{\circ} \mathrm{C}(\boldsymbol{\square}$; liquid), $25^{\circ} \mathrm{C}$ (*; gel) and $43^{\circ} \mathrm{C}(\square$; gel). Inset: Temperature dependence of the peak intensity $I_{\text {peak }}$. Line is drawn to guide the eye.

crowded solution (6.44 wt\%) the effect of temperature is virtually negligible in both liquid and gel state. Here, the increased excluded volume results in very little, if any, structural variation. This is more reminiscent of hard sphere behavior undergoing a glass transition [1]. These findings suggest a stronger interpenetration in the region $\phi<\phi_{c}$ and jamming in the region $\phi>\phi_{c}$, providing further support for the structural distinction between temperature-induced and concentration-induced gelation. To rationalize this finding, we argue that the weak gel (Fig. 8) has a broader size and distance distribution than the fluid, due to the various clusters coexisting with the single stars $[19,20]$. Hence, the peak of $I(q)$ is weaker for the gel than for the fluid; this is also evident from the experimental data, by comparing the peak intensities at roughly the same temperature (about $45^{\circ} \mathrm{C}$ ) for two concentrations in the liquid (higher) and the gel (lower) regions (insets of Figs. 8 and 9). Note that this effect is not qualitatively changed by the much smaller decrease of the form factor due to swelling, as evidenced by SANS measurements in other multiarm stars $[18,20]$. On the other hand, in the strong gel state (Fig. 9) the stars are practically frozen (jammed) in their instantaneous positions held already from the fluid configuration, and hence the $I(q)$ is unchanged; here, the effect of very weak swelling and jamming might imply that the $S(q)$ of the gel has an even higher peak value than that of the liquid. This again explains the large difference in the values of $G_{g e l}^{\prime}\left(T_{g e l}\right)$ for the two cases discussed with respect to Figs. 7-9. Furthermore, dynamic light scattering studies indicate that whereas the weak gels are ergodic $[19,20]$, the strong gels are clearly nonergodic [40]. Finally, it is noted that in a recent study of aqueous poly(vinyl alcohol) gels, the distinct difference between temperatureinduced and concentration-induced gelation was manifested 
as a continuous change versus divergence, respectively, of the correlation length at the transition [41]. The sharp drop of $V_{\text {int }}$ at $\phi_{c}$ in the inset of Fig. 7, signifying the concentrationinduced gel region, conforms to such a description.

In the absence of attractive interactions, the star arm expansion upon heating and the related excluded volume interactions are responsible for the slow formation of long lived clusters $[19,20]$, which kinetically arrest the system and form a gel, much like a glass formation process [1,7-9,42-44]. This work provides solid experimental evidence that this behavior is rather generic for the class of materials characterized by ultrasoft repulsions. Several applications of this effect can be envisaged in colloid science (e.g., stabilization, viscosity enhancement), as well as in processing of materials at nanoscopic dimensions (formation of templates, trapping of particles in tunable gel matrices). The fact that solvent quality (and thus temperature) affects the rheological properties through the change of the effective size and thus volume fraction, is already known $[45,46]$; the additional requirement for a kinetic trapping is the large softness (defined as the ratio of the corona to the overall radius, and typically assuming values of 0.9 ), which translates into significant volume fraction changes and excluded volume effects, giving rise to the temperature-induced gelation.

\section{CONCLUDING REMARKS}

In this work the dynamics of crowded solutions of multiarm star polymers suspended in moderate solvents was investigated as function of temperature, concentration and solvent quality. We found that these systems, which represent model ultrasoft colloidal spheres, undergo a reversible gelation upon heating. This phenomenon was attributed to the slow formation of clusters causing a kinetic arrest of the swollen interpenetrating stars at high temperatures, bearing analogies to the colloidal glass transition. We demonstrated that the choice of the solvent affects a great deal the gelation transition, as the solvent quality relates to the degree of star swelling. The requirement for the glass-like gelation is a high volume fraction, and as such for the same star concentration, a reduced quality of solvent implied higher gelation temperature (alternatively, for the same temperature, reduced solvent quality called for higher gelation concentration). A generalized description of this type of dynamic frustration was reflected in the kinetic pseudophase diagram for the gelation of the stars in different solvents, which indicated that a critical "soft sphere close packing" volume fraction $\phi_{c}$ exists for each solvent, distinguishing the temperature-induced (for $\phi$ $<\phi_{c}$ ) from the concentration-induced (for $\phi>\phi_{c}$ ) glass-like gelation. Therefore, appropriate choice of the solvent opens up a wide range of possibilities for the molecular manipulation of the sol-gel transition in such ultrasoft colloids.

\section{ACKNOWLEDGMENTS}

J.R. would like to acknowledge the hospitality of FORTH in Heraklion during his 2000-2001 sabbatical leave. Partial support from the EU (Grant No. HPRN-CT-2000-00017) and the Greek Ministry of Education (Grant No. 1090-Applied Molecular Spectroscopy) is gratefully acknowledged. We would like to thank C.N. Likos for helpful discussions and comments.
[1] Soft and Fragile Matter. Nonequilibrium Dynamics, Metastability and Flow, edited by M. E. Cates and M. R. Evans (Institute of Physics Publishing, Bristol, 2000).

[2] P.N. Segrè et al., Phys. Rev. Lett. 86, 6042 (2001).

[3] W. B. Russel et al., Colloidal Dispersions (Cambridge University Press, New York, 1989).

[4] P.N. Pusey and W. van Megen, Phys. Rev. Lett. 59, 2083 (1987).

[5] W. van Megen and S.M. Underwood, Phys. Rev. E 49, 4206 (1994)

[6] K.N. Pham et al., Science 296, 104 (2002).

[7] A.M. Puertas et al., Phys. Rev. Lett. 88, 098301 (2002).

[8] V. Trappe et al., Nature (London) 411, 772 (2001).

[9] Jamming and Rheology: Constrained Dynamics on Microscopic and Macroscopic Scales, edited by A. J. Liu and S. R. Nagel (Taylor and Francis, New York, 2001).

[10] P.A. Nommensen et al., Phys. Rev. E 59, 3147 (1999).

[11] J.C. Castaing et al., Europhys. Lett. 36, 153 (1996).

[12] M. Daoud and J.P. Cotton, J. Phys. (Paris) 43, 531 (1982).

[13] D. Vlassopoulos et al., J. Phys.: Condens. Matter 13, R855 (2001).

[14] C.N. Likos et al., Phys. Rev. Lett. 80, 4450 (1998).

[15] R. Borrega et al., Europhys. Lett. 47, 729 (1999).

[16] H. Senff et al., Langmuir 15, 102 (1999).
[17] J. Roovers, Macromolecules 27, 5359 (1994).

[18] C.N. Likos et al., Phys. Rev. E 58, 6299 (1998).

[19] M. Kapnistos et al., Phys. Rev. Lett. 85, 4072 (2000).

[20] B. Loppinet et al., Macromolecules 34, 8216 (2001).

[21] A.P. Gast and W.B. Russel, Phys. Today 51(12), 24 (1998).

[22] J. Mewis and J. Vermant, Prog. Org. Coat. 40, 111 (2000).

[23] T. Sato et al., Macromolecules 33, 1686 (2000).

[24] J. Buitenhuis and S. Förster, J. Chem. Phys. 107, 262 (1997).

[25] J.M. Sebastian et al., Macromolecules 35, 2700 (2002).

[26] J.S. Shay et al., J. Rheol. 45, 913 (2001).

[27] J. Roovers et al., Macromolecules 26, 4324 (1993).

[28] G.S. Grest et al., Adv. Chem. Phys. XCIV, 67 (1996).

[29] D. Richter et al., J. Phys. IV 8, 3 (1993).

[30] R. Berne and R. Pecora, Dynamic Light Scattering (Wiley, New York, 1976).

[31] J.F. Douglas et al., Macromolecules 23, 4168 (1990).

[32] L. Willner et al., Macromolecules 27, 3821 (1994).

[33] W.D. Dozier et al., Macromolecules 24, 2810 (1991).

[34] C.M. Marques et al., Eur. Phys. J. B 3, 353 (1998).

[35] H.H. Winter and M. Mours, Adv. Polym. Sci. 134, 165 (1997).

[36] M.C. Grant and W.B. Russel, Phys. Rev. E 47, 2606 (1993).

[37] V. Trappe and D.A. Weitz, Phys. Rev. Lett. 85, 449 (2002).

[38] J.A. Yanez et al., J. Colloid Interface Sci. 209, 169 (1999).

[39] A.N. Semenov et al., Langmuir 15, 358 (1999). 
[40] M. Stiakakis et al. (unpublished).

[41] M. Shibayama et al., Macromolecules 33, 7868 (2000).

[42] K. Dawson et al., Phys. Rev. E 63, 011401 (2000).

[43] K.A. Dawson, Curr. Opin. Colloid Interface Sci. 7, 218 (2002).
[44] W. Härtl, Curr. Opin. Colloid Interface Sci. 6, 479 (2001).

[45] M.D. Croucher and T.H. Milkie, Faraday Discuss. Chem. Soc. 76, 261 (1983).

[46] J. Mewis et al., AIChE J. 35, 415 (1989). 